\title{
Invertible Watermarking Algorithm with Detecting Locations of Malicious Manipulation for Biometric Image Authentication
}

\author{
Jaehyuck Lim ${ }^{2}$, Hyobin Lee ${ }^{1}$, Sangyoun Lee ${ }^{2}$, and Jaihie Kim² \\ ${ }^{1}$ Biometric Engineering Research Center (BERC), \\ Graduate Program in Biometrics \\ ${ }^{2}$ Department of Electrical and Electronic Engineering, Yonsei University, \\ 134, Shinchon-dong, Seodaemon-ku, Seoul, 120-749, Korea \\ Tel:+82-2-2123-5768, Fax:+82-2-362-5563 \\ \{jhlim, leehb00, syleee, jhkim\}@yonsei.ac.kr
}

\begin{abstract}
In this paper, we present a new method for authentication of biometric images. Our method uses an invertible watermark that can also detect malicious manipulations simultaneously. While virtually all watermarking schemes introduce a small amount of non-invertible distortion in original biometric images, our new method is invertible in the sense that, if the data is deemed authentic, distortion due to authentication can be removed if it becomes necessary to obtain the original biometric image. This technique provides cryptographic strength when verifying image integrity because the probability of making an undetectable modification to the image can be directly related to a secure cryptographic element, such as a hash function. Also, if the biometric image is manipulated, the positions of intentional manipulation can be clearly identified.
\end{abstract}

\section{Introduction}

With the present widespread utilization of biometric identification systems, establishing the authentication of biometric images (face, fingerprint, iris etc.) themselves has emerged as an important research topic. Cryptography and digital watermarking techniques are two possible ways of achieving this. While cryptography focuses on methods of making encrypted information meaningless to unauthorized parties [1], digital watermarking techniques can be used to embed proprietary information in host biometric images in order to protect the intellectual property rights and authentication of those images [2]. Encrypted templates are secure since they cannot be utilized or modified without decrypting them with the correct key, which is typically secret. But cryptography does not provide any security once data has been decrypted. Since there is a possibility that decrypted data can be intercepted, cryptography does not address the overall security of biometric images. However, since digital watermarking involves embedding information directly into host biometric images, these images are secure even after decryption. The watermark, which is not related to encryptiondecryption operations, provides another line of defense against the illegal utilization of biometric images. 
One possible drawback of authentication based on watermarking is that the authenticated biometric image will inevitably be distorted by a small amount of noise due to authentication itself [3]. In virtually all previously proposed watermarking schemes, this distortion cannot be completely removed even when the image is deemed authentic. Although the distortion is often quite small, it may be unacceptable in certain kinds of biometric images, for example, iris and fingerprint images. In this paper, we analyze the conditions under which it is possible to "undo" changes introduced by authentication if the biometric image is verified as authentic. We present techniques that make this kind of invertible authentication possible. Then, we embed an invertible message authentication code into the biometric image so that anyone who possesses the authentication key can revert to an exact copy of the original biometric image that existed before authentication occurred. If the biometric image is also manipulated, the proposed method can protect the biometric contents from malicious manipulation and detect modified parts of the biometric data at the same time.

The rest of this paper is organized as follows. In section 2, we explain the proposed invertible watermarking algorithm. Simulation results are given in section 3 . Finally, the main contributions of this work and suggestions for future research are summarized in section 4 .

\section{The Proposed Watermarking Algorithm}

Figure 1 shows the overall structure of our proposed invertible watermarking algorithm. The algorithm is able to authenticate biometric images as well as detect manipulated parts. The proposed watermark embedding consists of two stages: invertible biometric image authentication and detection of intentional biometric image attacks. These two stages are explained in more detail in the following subsections.

\subsection{Embedding Watermark for the Authentication and Invertibility of the Biometric Image}

For the authentication and invertibility of the biometric image, we applied the conventional image watermarking method to the biometric image [3]. Let us assume that the original biometric image is a grayscale image with pixel values from the set $P=\{0$, $\ldots, 255\}$. We started to calculate the hash value of the biometric image which consists of 128 bits. In our experiments, we used the MD5 hash function. After that, we divided the image into disjoint groups of $n$ adjacent pixels $\left(\mathrm{x}_{1}, \mathrm{x}_{2}, \ldots, \mathrm{x}_{n}\right)$. We also defined the so-called discrimination function $f$ that assigns a real number $f\left(\mathrm{x}_{1}, \mathrm{x}_{2}, \ldots, \mathrm{x}_{n}\right)$ to each disjoint group $G=\left(\mathrm{x}_{1}, \mathrm{x}_{2}, \ldots, \mathrm{x}_{n}\right)$ as follows.

$$
f\left(G=\left(x_{1}, x_{2}, \ldots, x_{n}\right)\right)=\sum_{i=1}^{n-1}\left|x_{i+1}-x_{i}\right|
$$

Then, we defined an invertible operation as $F$ on $P$. This operation is known as flipping and features a permutation of gray levels that entirely consists of two cycles. Thus, $F$ has the properties of $F^{2}=$ Identity or $F(F(x))=x$ for all $x \in P$. We used the discrimination function $f$ and the flipping operation $F$ to define three pixel groups: regular $(R)$, singular $(S)$, and unusable $(U)$. 


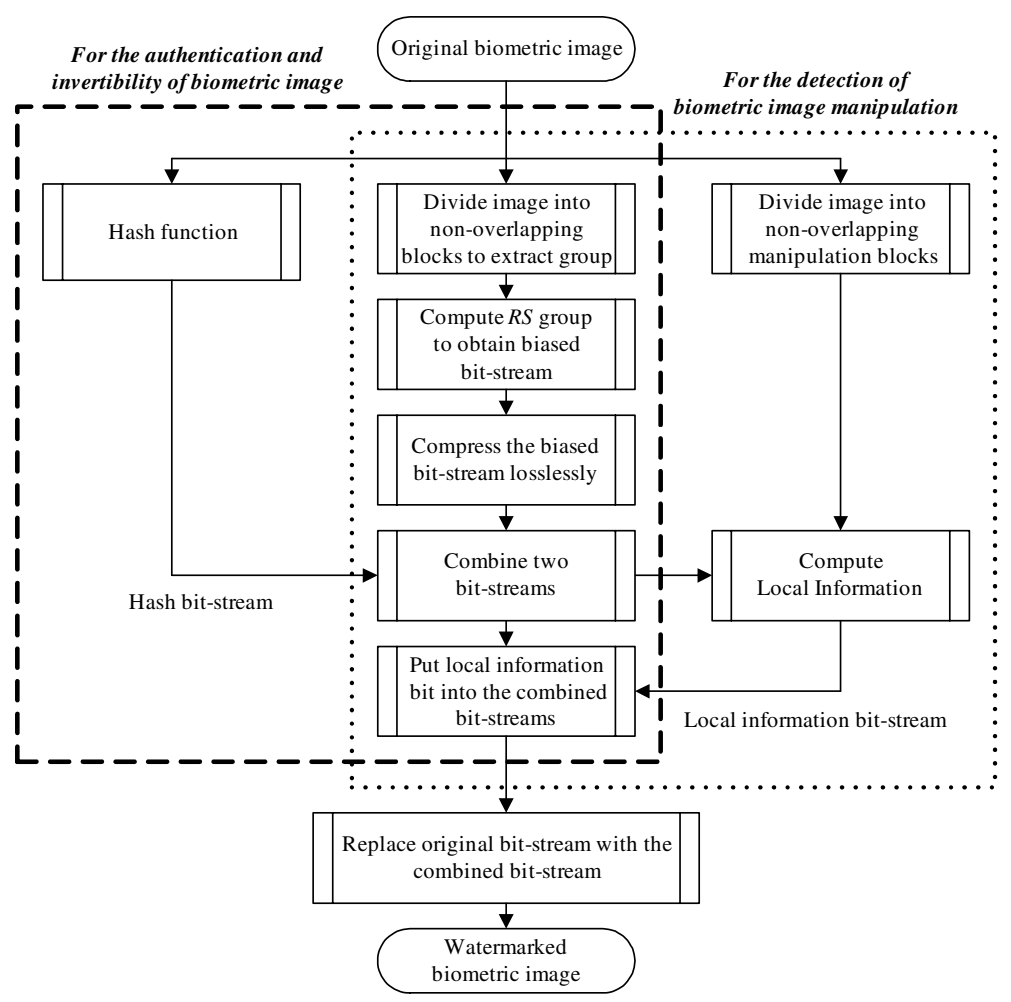

Fig. 1. Flowchart of the proposed algorithm

$\begin{array}{lll}\text { Regular groups: } & G \in R \Gamma \text { if } & f(F(G))>f(G) \\ \text { Singular groups: } & G \in S \quad & \text { if } f(F(G))<f(G) \\ \text { Unusable groups: } & G \in U\ulcorner\text { if } & f(F(G))=f(G) .\end{array}$

Thus, the $R$ and $S$ groups are flipped into each other under the flipping operation $F$, while the unusable groups $U$ do not change their status. In a symbolic form, $F(R)=S$, $F(S)=R$, and $F(U)=U$. We then formulated the results using the data embedding method. By assigning a 1 to $R$ and a 0 to $S$, we embedded one message bit in each $R$ or $S$ group. The $R S$ bit-stream normally has a large number of ' 0 ' or ' 1 '. So, to reduce the data, we adopted the context-free lossless compression algorithm for the $R S$ bitstream in order to obtain the compressed $R S$ bit-stream. So, after lossless compression, the length of the bit-stream was reduced significantly. Namely, the compression created an empty space that was used to store the hash bit-stream, which provided authentication, and the local information bit-stream, which detected malicious manipulation. Details of the local information and embedding process are discussed in the next subsection. 


\subsection{Embedding Watermarks for Detection of Malicious Manipulation}

Details of the algorithm are as follows. First, as explained in section 2.1, the 128-bit hash bit-stream was computed from the original biometric image as shown in Eq. (3), and the biased $R S$ bit-stream was also obtained as shown in Eq. (4).

$$
\begin{aligned}
& \text { Hash bit-stream }=\left\{H_{k} \in\{0,1\} \mid 1 \leq k \leq 128\right\} \\
& R S \text { bit-stream }=\left\{R S_{i j} \in\{0,1\} \mid 1 \leq i \leq I, 1 \leq j \leq J\right\}
\end{aligned}
$$

In Eq. (4), $J$ is the number of non-overlapped manipulation blocks obtained from the original biometric image, and $I$ is the number of $R S$ groups in each block. Next, lossless compression was performed on the obtained $R S$ bit stream. The compression created an empty space that was used to store the hash bit stream already obtained from the original biometric image. This made it possible to authenticate the watermarked image and to store the local information bit-stream to detect the location of malicious manipulations in the attacked image. When the corresponding bit-stream was put into the space, a user-defined bit-stream shuffling method was used to increase the security level of the proposed algorithm. As a result, the combined bitstream was obtained by concatenating and shuffling the compressed $R S$ bit-stream and the hash bit-stream as shown in Eq. (5). The size of the combined bit-stream is designed to be equal to that of the $R S$ group. In the combined bit-stream, the last bit of each manipulation block, $C R S_{I j}$, was left empty to store local information. To obtain the combined bit-stream with local information bits, a user-defined sequence with size of $J$ was generated as shown in Eq. (6).

$$
\begin{aligned}
& \text { Combined bit-stream }=\left\{C R S_{i j} \in\{0,1\} \mid 1 \leq i \leq I, 1 \leq j \leq J\right\} \\
& \text { User-defined sequence }=\left\{P_{j} \in\{0,1\} \mid 1 \leq j \leq J\right\}
\end{aligned}
$$

$J$ is the number of blocks used to detect the manipulated location. All bits of each manipulation block excluding the last local information bit were summed. Then, the local information bit was assigned to equalize the LSB of the summed value and the corresponding bit of the user-defined sequence as shown in Eq. (7).

$$
\begin{aligned}
& \text { Local information } L_{j}= \begin{cases}1 & \text { if }\left(\left(\sum_{i=1}^{I-1} C R S_{i j}\right) \% 2\right) \neq P_{j} \\
0 & \text { if }\left(\left(\sum_{i=1}^{I-1} C R S_{i j}\right) \% 2\right)=P_{j}\end{cases} \\
& \text { Watermark embedding }\left\{\begin{array}{ccc}
F\left(R S_{i j}\right) & \text { if } & C R S_{i j} \neq R S_{i j} \\
\text { skip } & \text { if } & C R S_{i j}=R S_{i j}
\end{array}\right.
\end{aligned}
$$

Finally, the $C R S_{i j}$ bit stream assigned local information bit was put into the $R S$ group as shown in Eq. (8). This means that when both the $R S_{i j}$ and $C R S_{i j}$ were compared, if the two values were the same, $R S_{i j}$ was used as the bit-stream, otherwise the $R S$ group mode was flipped according to function $F$, defined in Section 2.1. When the watermarked image is manipulated, the $R(S)$ group may be converted to the $S(R)$ group, or local information bit may be changed. Based on this information, if the 
number of 1 s of the $R$ group in each manipulation block is different from the generated user-defined sequence as shown in Eq. (6), the block is assumed to have been manipulated. Then, the proposed algorithm can only check the LSB of the number of 1s in each block.

\subsection{Integrity and Manipulation Verification}

Figure 2 shows the overall structure of our watermark detection process. The user extracts the bit-streams from all $R$ and $S$ groups $(R=1, S=0)$ by scanning the watermarked biometric images in the same order as they were scanned during embedding. The extracted bit-stream is separated into the embedded original hash bitstream, the embedded original local information bit-stream, and the embedded compressed $R S$ bit-stream. The compressed $R S$ bit-stream is decompressed to reveal the original status of all the $R$ and $S$ groups. The image is then processed once more and the status of all the groups is adjusted as necessary by flipping them back to their original states. Thus, exact copies of the original images are obtained. Then, we calculate the hash values of the restored images and compare the calculated hash values with the embedded hash ones. If the two hash values are identical, the biometric image is authentic. This means that the restored biometric images can be verified to be the original ones. Otherwise, we check the relationship between the number $1 \mathrm{~s}$ of each manipulation block and of the corresponding local information bit. If they are different, the corresponding blocks are shown to have been manipulated intentionally.

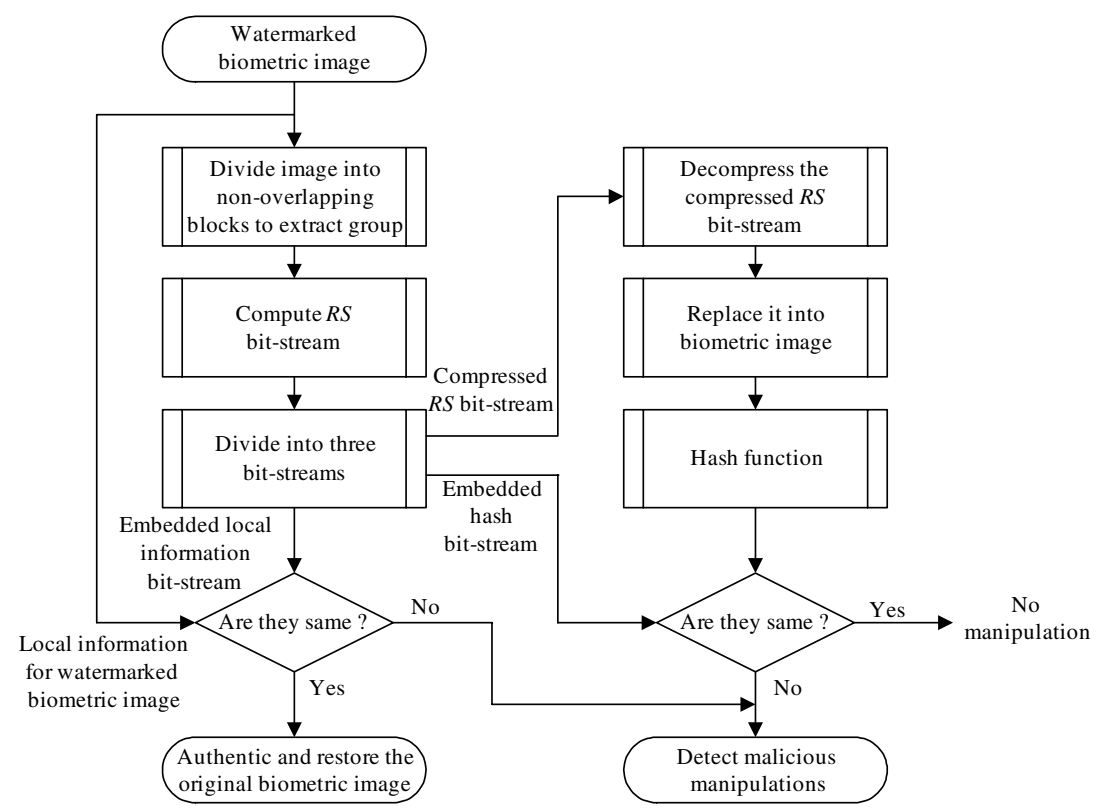

Fig. 2. The watermark detection process 


\section{Experimental Results}

To demonstrate the proposed biometric image authenticator, we used several images in our experiments (face, fingerprint, and iris), as shown in Fig. 3. The corresponding watermarked images are shown in Fig. 4. As can be seen, distortion due to watermarking is invisible and can be removed from the watermarked image if it is deemed authentic. For the detection of malicious manipulation, the watermarked biometric images in Fig. 4 were manipulated partly (in case of face and iris images) and were

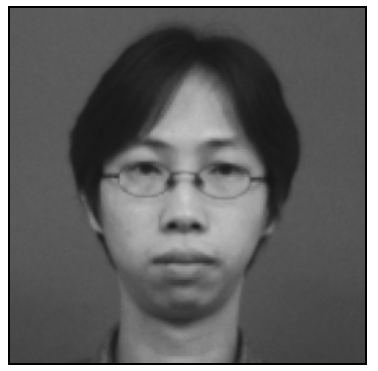

(a) Face image

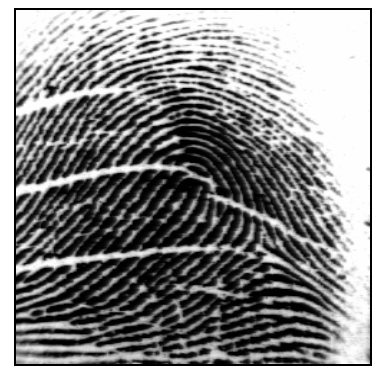

(b) Fingerprint image

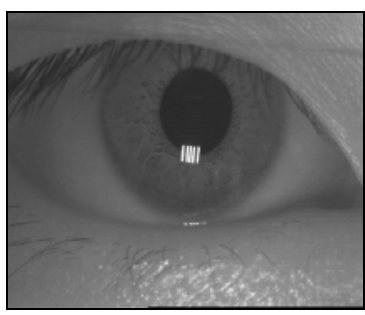

(c) Iris image

Fig. 3. Original biometric images

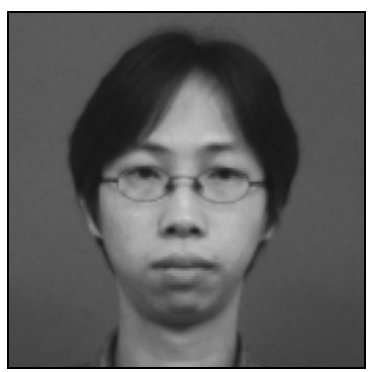

(a) Face image

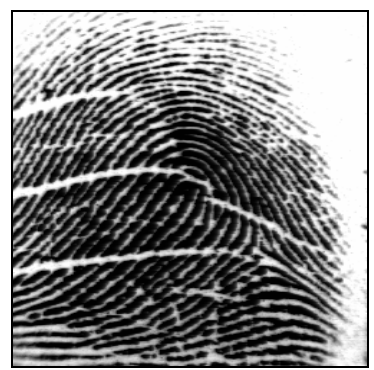

(b) Fingerprint image



(c) Iris image

Fig. 4. Watermarked biometric images

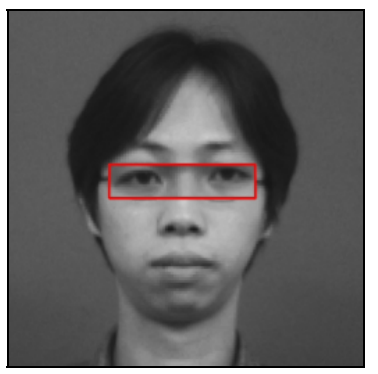

(a) Face image

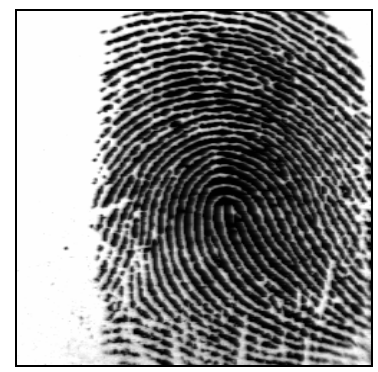

(b) Fingerprint image

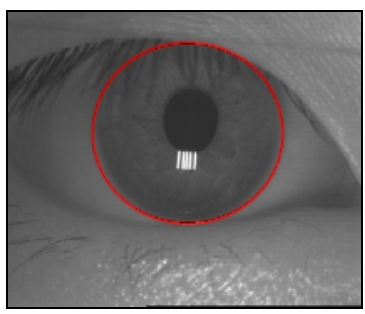

(c) Iris image

Fig. 5. Manipulated biometric images 


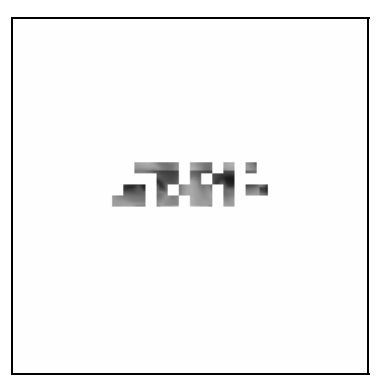

(a) Face image

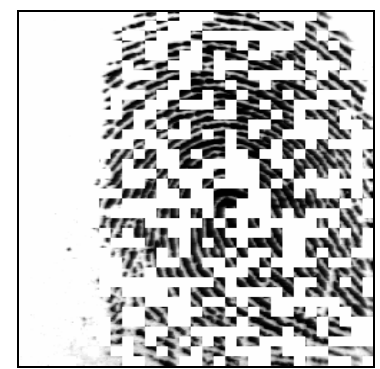

(b) Fingerprint image

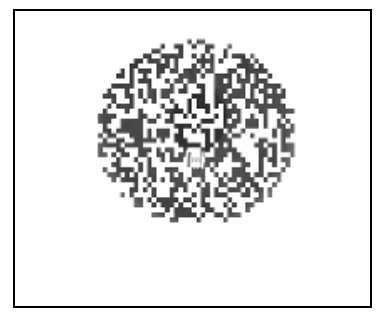

(c) Iris image

Fig. 6. Block-based detection of the manipulated biometric images in Fig. 5

modified by content-based manipulations (in case of fingerprint images) as shown in Fig. 5. Applying the proposed algorithm, we were able to identify the block-based locations of the intentional manipulations as shown in Fig. 6.

\section{Conclusions}

In the paper, we proposed an invertible biometric image watermarking algorithm which can also locate the positions of intentionally manipulated blocks. While virtually all watermarking schemes introduce a small amount of non-invertible distortion in original biometric images, our new proposed method is invertible in the sense that, if the image is deemed authentic, distortion due to authentication can be removed to obtain the original biometric image. Also, if the biometric image is manipulated, the positions of the intentional manipulation can be identified.

\section{Acknowledgements}

This work was supported by the Korea Science and Engineering Foundation (KOSEF) through the Biometrics Engineering Research Center (BERC) at Yonsei University.

\section{References}

1. O. Khalifa, M. Islam, S. Khan, M. Shebani, "Communications cryptography", RF and Microwave Conference, 2004. RFM 2004. Proceedings, 5-6 Oct. pp. 220-223, 2004.

2. A. Kejariwal, "Watermarking", Potentials, IEEE, vol. 22, Issue 4, pp. 37-40, Oct.-Nov. 2003.

3. J. Fridrich, M. Goljan, R. Du, "Invertible authentication watermark for JPEG images", International Conference on Information Technology: Coding and Computing (ITCC), Las Vegas, Nevada, USA, pp. 223-227, April 2001. 